\title{
Quantitative proteomic analyses on the mechanisms of cold tolerance in two peach cultivars (Prunus persica L. Batsch) based on ITRAQ
}

\author{
Yonghong Li ${ }^{1, *}$, Zhaoyuan Wang ${ }^{1}$, Qihang Tian ${ }^{1}$, Yuchao Zhou ${ }^{2}$, Jintao $\mathrm{Xu}^{1}$, Ruifeng Chang ${ }^{1}$, Hu Chen ${ }^{1}$ \\ and Guojian Liu ${ }^{1}$ \\ ${ }^{1}$ Changli Fruit Institute, Hebei Academy of Agriculture and Forestry Sciences, Changli, Hebei, PR China \\ ${ }^{2}$ Hebei Academy of Agriculture and Forestry Sciences, Shijiazhuang, Hebei, PR China
}

\section{Summary}

Cold tolerance is the main feature of life activity in Prunus persica, which directly affects the introduction and breeding for resistance. However, the molecular mechanisms underlying this process remain unknown. Therefore, isobaric tag for relative and absolute quantification (iTRAQ)-based comparative proteomic analysis was conducted to identify cold-responsive proteins in cold-tolerant peach cultivar (CT) and cold-sensitive peach cultivar (CS) in response to cold stress. This study examined 2,575 different proteins, among which, 322 and 399 showed significantly differential accumulation within CS and CT peach cultivars, respectively, following cold stress for $\mathbf{4 8} \mathrm{h}$. As suggested by bioinformatic analyses, compared with CS, CT potentially displayed a relationship with the greater amount of energy generated from carbon metabolism, starch and sucrose metabolism, and phenylpropanoid biosynthesis to resist cold stress. Peroxidase, flavonoid, carbonic anhydrase, and harpin proteins were more abundant in CT, which suggested that they might play roles in mitigating the cold resistance-induced damage. Besides, a variety of strategies were utilized to respond to cold stress in CT and CS, due to the different pathogenesis-related (PR) proteins. In addition, real-time PCR (RT-PCR) indicated that five candidate protein-encoding genes (M5WUF0, M5W9D5, H9EBJ3, Q5QJB3, and M5W6Z4) were highly expressed, which were consistent with iTRAQ data in CT. Taken together, our results suggest that five genes are involved in modulating the cold stress mechanisms of CT. Our study provides useful information for understanding the underlying mechanisms of cold tolerance and identifying the candidate resistant proteins in peach, which possibly facilitates to breed peach cultivars with enhanced cold tolerance.

\section{Keywords}

Prunus persica, cold-tolerance, cold-sensitive, proteomics, cold stress

\section{Introduction}

Peach (Prunus persica L. Batsch), a nutrient-rich fruit, has been extensively distributed worldwide (Wu et al., 2017); however, the low temperature is a critical limiting factor for its growth, development, and distribution (Niu et al., 2020).

\section{Significance of this study}

What is already known on this subject?

- The proteomic analyses of numerous plant species in response to cold have been well-investigated, such as grape, watermelon, winter turnip rape, wheat, cucumber, and Petunia. However, it remains unclear how the proteomics of peach trees respond to cold stress, and key proteins involved in cold tolerance of peach trees are rarely reported. Therefore, it is essential to understand the mechanism of cold tolerance in peach at proteome level.

What are the new findings?

- First, we have found that the cold-tolerant peach cultivar was related to carbon metabolism, starch as well as sucrose metabolism, and phenylpropanoid biosynthesis. Second, peroxidase, flavonoid, carbonic anhydrase, and harpin proteins play important roles in cold tolerance of the cold-tolerant peach cultivar. Third, ten candidate protein-encoding genes were validated in RNA level by real-time PCR.

What is the expected impact on horticulture?

- Cold resistance is the main characteristic of life activity in fruit trees, which directly affects the introduction and resistance breeding. Peach is an extensively distributed fruit plant worldwide, which shows significant heterogeneity in terms of cold resistance among various cultivars. Therefore, it is useful to examine the underlying mechanisms of cold resistance of peach, so as to breed the cultivars with enhanced cold tolerance, and to offer related proteomic information.

Generally, peach varieties with tolerance to low temperature better adapt to the low temperature, which may be potentially used to breed the cold-tolerant peach cultivars as the important germplasm resources in traditional breeding methods. However, little is known about the improvement in the cold tolerance mechanism of peach at the molecular level. Consequently, it is helpful to illustrate the biochemical, physiological, and protein changes in peach in the presence of cold stress, which contributes to enhancing the cold tolerance of peach cultivars through genetic engineering.

Prior studies are mostly carried out to examine the peach cytology, biochemistry, morphology, genetics, and physiology, together with the cold resistance mechanisms (Howell et 
al., 1997; Marafon et al., 2009; Nilo-Poyanco et al., 2019; Tareen et al., 2017). The proteomic analyses of numerous plant species in response to cold have been well-investigated, such as grape (Deng et al., 2017), watermelon (Shi et al., 2019), winter turnip rape (Xu et al., 2018), wheat (Zhang et al., 2018), cucumber (Fan et al., 2015), and Petunia (Zhang et al., 2016). However, it remains unclear about how the proteomics of peach trees responds to cold stress, and key proteins involved in cold tolerance of peach trees are rarely reported. Therefore, it is essential to understand the mechanism of cold tolerance in peach at proteome level.

Compared with conventional genomic analysis, a proteomic analysis aims to directly depict responses at the molecular level, which sheds more light on the immunity of a plant. Isobaric tags for relative and absolute quantification (iTRAQ) are employed to carry out quantified proteomic analysis on several samples based on singular sensitive mass spectrometry (MS) (Chen et al., 2013; Wang et al., 2016). Such an approach contributes to accurately assessing and quantifying the protein contents, in the meantime of reducing the individual experimental errors (Chen et al., 2013). It has been verified that proteomic analysis is an efficient approach to study the cold stress tolerance and response mechanisms, which has been carried out among various plants ( $\mathrm{Su}$ et al., 2016; Chen et al., 2016).

The aim of this work was to carry out comparative proteomic analyses based on ITRAQ with the final goal of identifying the cold-respondent proteins in cold-tolerant and cold-sensitive peach cultivars.

\section{Materials and methods}

\section{Plant materials}

Trials were performed within the peach orchard in Changli of China $\left(39^{\circ} 45^{\prime} \mathrm{N}, 119^{\circ} 12^{\prime} \mathrm{E}\right)$. In this study, two peach cultivars were adopted, namely, Prunus persica Batsch cv. 'Cold Princess' (CT, cold-tolerant) and Prunus persica Batsch cv. ' $21^{\text {th }}$ Century' (CS, cold-sensitive) cultivars. Trees were planted at the north-south orientation at the spacing of $2 \mathrm{~m} \times 4 \mathrm{~m}$. There were sandy loam soils in our experimental site, which were beneficial for a favorable management and the irrigation system.

\section{Cold treatments}

Forty healthy annual branches at equivalent length (30$35 \mathrm{~cm}$ ) and diameter $(1.0 \mathrm{~cm})$ were collected from all sides of the eight-year-old tree on December $28^{\text {th }}, 2018$. The branches were then washed with distilled water to clean the dust and impurities, and dried with the clean gauze. Later, each cultivar was divided into three parts, among which, one was used as the control material, whereas the other two were placed in the growth chamber, and the temperatures were reduced to the predetermined temperature at $0^{\circ} \mathrm{C}$ and $-25^{\circ} \mathrm{C}$ for $48 \mathrm{~h}$, respectively. Each treatment was repeated for three times. The temperatures were maintained for $48 \mathrm{~h}$ after reaching the required values, and the cooling rates were set at $3^{\circ} \mathrm{Ch}^{-1}$.

\section{Measurements of REL and GR}

After applying the low-temperature treatment, samples were thawed at $-4^{\circ} \mathrm{C}$ for $24 \mathrm{~h}$ in the refrigerator, and then preserved at room temperature for $12 \mathrm{~h}$. The relative electrolyte leakage (REL) of those treated branches was determined based on Yun et al. (2014)'s method. The $0^{\circ} \mathrm{C}$ (T48) and $-25^{\circ} \mathrm{C}(\mathrm{T} 48)$ treated samples were collected, respectively. Later, all samples were immersed in liquid nitrogen imme- diately and preserved under $-80^{\circ} \mathrm{C}$ before use. Protein was extracted from $1 / 2$ samples, while the other $1 / 2$ were adopted to extract RNA.

Twenty days later, the germination number of treated branches was investigated. Afterward, both control and treated branches were put in water under the following conditions, light/dark cycle of $16 \mathrm{~h} / 8 \mathrm{~h}$, the temperature of $25^{\circ} \mathrm{C}$, and 5,000-6,000 lx. Then, the water was changed once a day, and the germination rate (GR) was investigated at 20 days later.

Germination rate $(\%)=$ germination number/total bud number $\times 100 \%$.

\section{Protein extraction}

Beijing Genome Institute (BGI) was responsible for implementing iTRAQ. The phenol extraction procedure was adopted to extract total proteins from the cortices of annual peach branches (Saravanan and Rose, 2004; Yao et al., 2006). Two repeated experiments were carried out for all samples.

\section{iTRAQ labeling as well as fractionation of strong cation exchange (SCX)}

The Bradford approach was employed to determine the protein contents (Wang et al., 2006). An equivalent amount of protein was collected for every repeated experiment. Specifically, the CS and CT protein samples were subjected to reduction using DTT (10 mM), alkylation using iodoacetamide $(55 \mathrm{mM}), 24 \mathrm{~h}$ digestion with trypsin at sequencing grade $\left(1: 10, w / w\right.$, Promega) under the temperature of $37^{\circ} \mathrm{C}$, and labeling with the iTRAQ 8-plex kits (AB Sciex Inc., MA, U.S.A.) in accordance with manufacturer instructions. As for controls, they were subjected to iTRAQ tags 115 and 116 (CK), 117 as well as 118 (cold treatments at $0^{\circ} \mathrm{C}$ ), 119 and 121 (cold treatments at $-25^{\circ} \mathrm{C}$ ) labeling, separately.

After labeling and quenching, each sample was integrated and freeze-dried, respectively. Then, SCX buffer A $(4 \mathrm{~mL}$, containing $10 \mathrm{mM} \mathrm{KH}_{2} \mathrm{PO}_{4}$ and $25 \% \mathrm{v} / \mathrm{v}$ acetonitrile, $\mathrm{pH} 3$ ) was adopted to dissolve peptide mixture, followed by fractionation using the Ultremex SCX column $(250 \times 4.60 \mathrm{~mm}$ $100 \AA$ ) on the Agilent 1100 HPLC system (Agilent, America). Afterward, elution buffer B supplemented with $10 \mathrm{mM}$ $\mathrm{KH}_{2} \mathrm{PO}_{4}, 2 \mathrm{M} \mathrm{KCL}$, and $25 \% \mathrm{v} / \mathrm{v}$ acetonitrile $(\mathrm{pH} 3$ ) was used to elute the peptides at $1 \mathrm{~mL} \mathrm{~min}^{-1}$. Later, the elution was observed through detecting the optical density at the wavelength of $214 \mathrm{~nm}$, and each fraction was extracted at an interval of 1 min. Afterward, those eluted peptides were integrated into 20 fractions, followed by desalting using the Strata X C18 column (Phenomenex, Torrance, CA, U.S.A.) as well as vacuum-drying before performing the subsequent LC-ESIMS/MS experiment.

\section{LC-MS/MS}

LC-MS/MS was carried out according to the previously described method (Liu et al., 2015; Chu et al., 2015). In brief, the peptide mixture after desalting was loaded on a reversed-phase C18 column (Acclaim PePmap C18, $75 \mu \mathrm{m} \times$ $2 \mathrm{~cm}, 3 \mu \mathrm{m}, 100 \AA ̊$, Thermo Scientific), followed by separation using the reversed-phase C18 column $(75 \mu \mathrm{m} \times 10 \mathrm{~cm}$, $5 \mu \mathrm{m}, 300 \AA$ Ag, Agela Technologies) equipped onto the Dionex ultimate 3000 nano LC system. After that, the Q-Exactive mass spectrometer (Thermo Fisher Scientific, MA, U.S.A.) was adopted for $5-80 \%$ acetonitrile (v/v) gradient elution within the $0.1 \%$ formic acid of the above peptide mixture

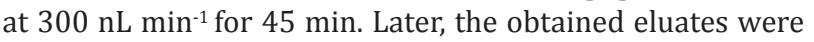


placed into the Q-Exactive MS (Thermo Fisher Scientific, Waltham, MA, U.S.A.) directly for analysis under the settings of data-dependent manner, positive ion mode, full scan resolution of 70,000, full MS scan of 350-2,000 m/z, along with the resolution, minimal signal threshold and isolation width of MS/MS scan of 17,500,1E+5, and $2 \mathrm{Da}$, respectively. To assess the MS performance on those samples labeled with iTRAQ the higher collision energy dissociation (HCD), together with two MS/MS acquisition modes, was adopted. In addition, the normalized collision energy (NCE) was evaluated systemically at 28 with the step of $20 \%$, to optimize the $\mathrm{HCD}$ acquisition efficiency in MS/MS.

\section{Real-time PCR was carried out for transcriptional validation}

After cold treatment for $48 \mathrm{~h}$, cortices from the annual peach branches (collected from samples under identical treatment for protein extraction) were collected to extract the total RNA through an RNA prep Pure Plant Kit (DP441, Tiangen, Beijing, China) in accordance with manufacturer instructions. Afterward, the RNA content was measured, its integrity was estimated, and cDNA was synthesized through reverse transcription of RNA, followed by real-time PCR (RT-PCR) as described previously (Li et al., 2015). In addition, all primers were prepared in accordance with relevant peach nucleotide sequences in GenBank. Table 1 shows the gene-specific primers used in RT-PCR.

\section{Bioinformatics analysis}

The Gene Ontology (GO) database (http://www.geneontology.org) was employed to perform functional annotations of the differently expressed proteins (DEPs) (Conesa et al., 2005). On the other hand, the primary pathways involved in metabolism were predicted using the Kyoto Encyclopedia of Genes and Genomes (KEGG) database (http://www.genome. jp/kegg/pathway.html) (Kanehisa et al., 2008).

\section{Statistical analysis}

The SPSS 20.0 software (SPSS Inc., Chicago, IL, U.S.A.) was adopted for the statistical analyses of all physiological and transcript data by the analysis of variance (ANOVA). All data were expressed in the manner of mean \pm standard error, and
TABLE 1. Primer sequences for RT-PCR analysis.

\begin{tabular}{llc}
\hline ID & Sequence (5'-3') & $\begin{array}{c}\text { Product } \\
\text { length (bp) }\end{array}$ \\
\hline P-actin. F & GGCTCAGTCTAAGCGTGGTA & 173 \\
P-actin. R & TGGCAGGAGTGTTGATGTC & \\
M5WUF0 F & CCCTACTACAACGTACCGCT & 110 \\
M5WUF0 R & ACCTGGATGAGCTGAGACAC & \\
M5W9D5 F & ACTTAGACCCCACAACTCCG & 227 \\
M5W9D5 R & CTCCCCATTACTTCCCACCA & \\
Q5QJB3 F & TCACTCTCTACATTCCTCGCC & 157 \\
Q5QJB3 R & TTCGAGAACAGAAACCTCGTC & \\
H9EBJ3 F & AGATGATGGTGTTGGCAGGA & 212 \\
H9EBJ3 R & GTCAGCATCCTCTTTGAGCG & \\
M5W6Z4 F & TGGTCATTGGACACAGTCGT & 154 \\
M5W6Z4 R & ATATCATGCCCACCCAGTCC & \\
M5WAW8 F & ATGCAACCAGTTTGGTGCTC & 163 \\
M5WAW8 R & GAATCCCCATTTGCCTGCC & \\
M5XCX2 F & ATCTTCCGCCCTGACAACTT & 169 \\
M5XCX2 R & CAAGTGAGTGGCAAACCTGA & \\
M5WC10.F & TGATGTGTCTGGAAACCGGA & 233 \\
M5WC10.R & CATCCTCCAACATTGCCTCG & \\
M5XNQ1 F & CTATCTGCCGAGTCATTGGC & 177 \\
M5XNQ1 R & ATCAGTGCAACCGACAAGG & 123 \\
M5VVU6 F & AGGCTGTGGCAGACATAGTT & \\
M5VVU6 R & AAATAAGAGACGGGCCCCAA & \\
\hline & & \\
\hline
\end{tabular}

the significance level was set at 0.05 . All experiments were performed in a completely randomized design with three replicates per sample.

\section{Results}

\section{REL and GR variations in CT as well as CS in respond to cold treatments}

After $48 \mathrm{~h}$ cold treatment, the REL of CT (30.50\%) and CS $(29.74 \%)$ was almost at the same level of $0^{\circ} \mathrm{C}$ (Figure 1A). However, the REL of CS increased to $50.50 \%$ compared with

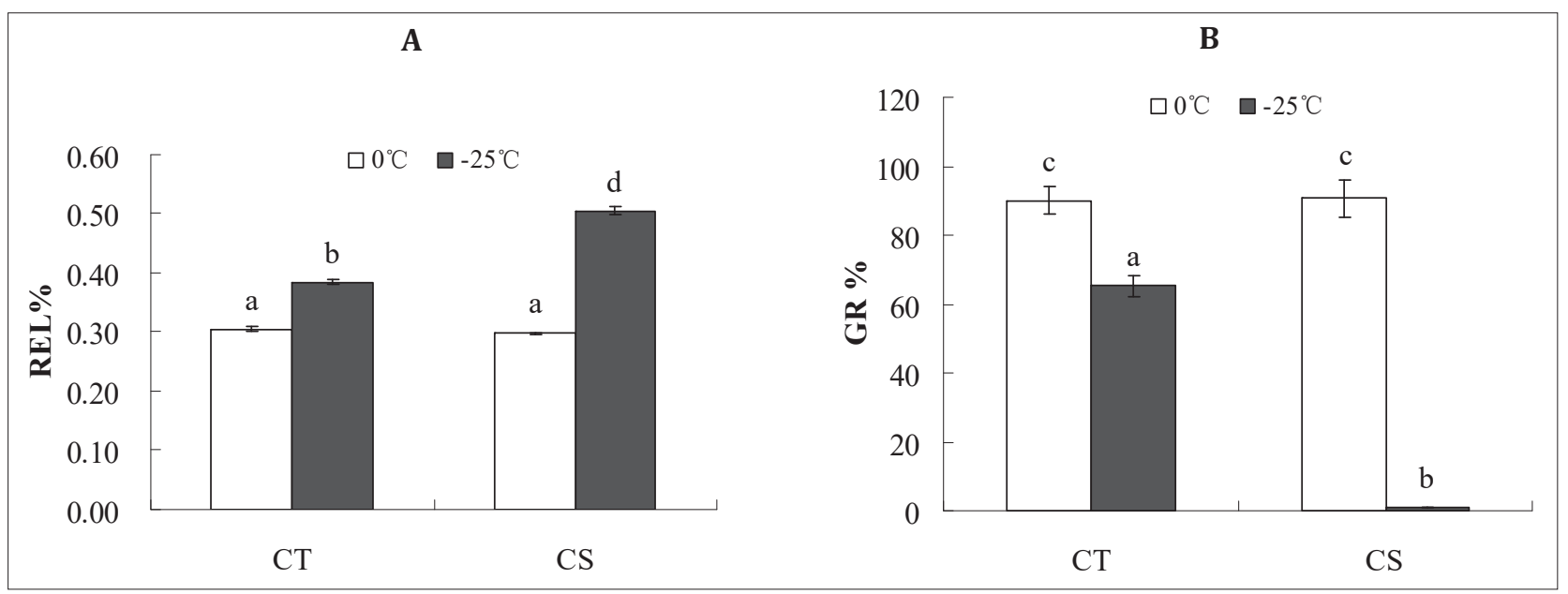

FIGURE 1. Physiological changes in cold-sensitive peach cultivar (CS) and cold-tolerant peach cultivar (CT) at cold stress conditions. (A) Relative electrolyte leakage (REL) \%. (B) Germination rate (GR) \% of CT and CS at $0^{\circ} \mathrm{C}$ for 48 h. The values were measured following $48 \mathrm{~h}$ cold stress treatment in plants, and were expressed in the manner of means \pm standard error $(n=3$ for GR and REL). The analysis of variance (ANOVA) approach was used to determine the significant differences (small letters, $\mathrm{P}<.05$ ). 

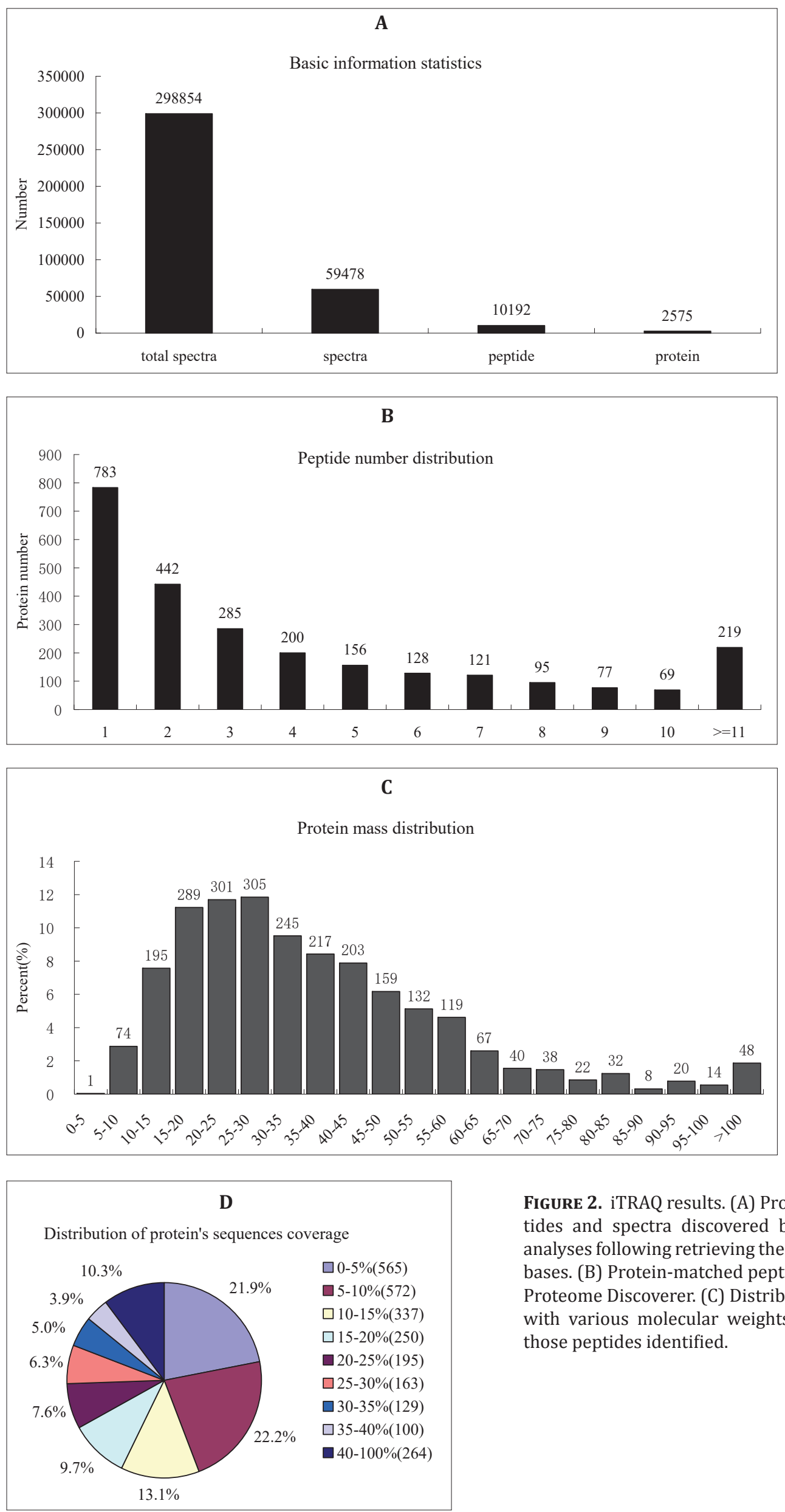

FIgURE 2. iTRAQ results. (A) Proteins, unique peptides, peptides and spectra discovered based on ITRAQ proteomic analyses following retrieving the Rosaceae family in the databases. (B) Protein-matched peptides number counted by the Proteome Discoverer. (C) Distributions of identified proteins with various molecular weights. (D) Protein coverage via those peptides identified. 


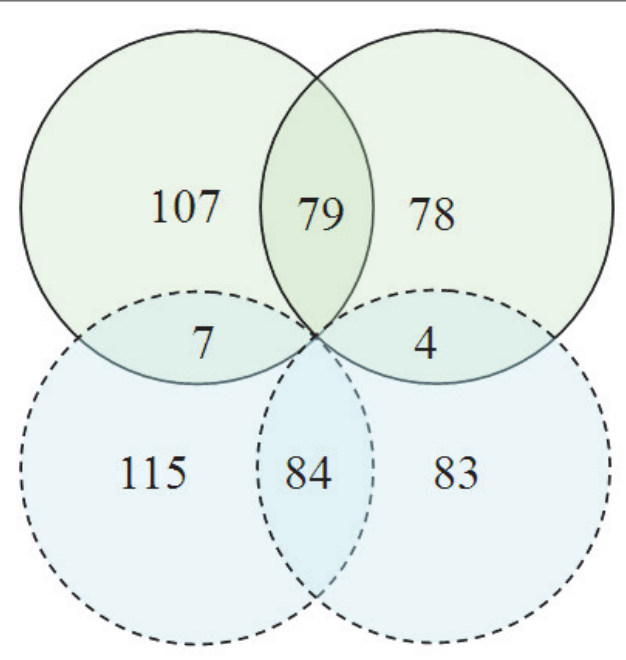

Up-regulated proteins in $\mathrm{CT}$

Up-regulated proteins in CT
Down-regulated proteins in CS

Down -regulated proteins in CS
FIGURE 3. Venn diagram showing the down-regulated and up-regulated proteins in CT as well as CS. CT and CS represented the cold-tolerant and cold-sensitive peach cultivars, respectively. that of $\mathrm{CT}$ at $-25^{\circ} \mathrm{C}(\mathrm{P}<0.05)$. As observed (Figure 1B), the GR of CT $(90 \%)$ and CS (90.67\%) was almost at the same level of $0^{\circ} \mathrm{C}$. However, the GR of CS decreased to $1 \%$ compared with that of CT $(65.33 \%)$ at $-25^{\circ} \mathrm{C}(\mathrm{P}<0.05)$. By contrast, the GR of CS remarkably decreased after cold stress at $-25^{\circ} \mathrm{C}$, but that was markedly higher in CT than in CS after cold stress at $-25^{\circ} \mathrm{C}$. In general, the low temperature had remarkably detrimental effects on the cold-sensitive peach cultivar.

\section{Analysis of primary data and detection of proteins}

Altogether 298,854 spectra were obtained based on iTRAQ tests on the cold-sensitive or cold-tolerant peach cortices as the raw materials. Later, the Proteome Discoverer (Mascot version 1.4) was used for data analysis. In total, 2,575 proteins and 10,192 peptides were identified based on those 59,478 spectra recognized via the Proteome Discoverer (Figure 2A, Supplemental Table S1). Figure 2B shows the peptide number distribution of every protein. As observed (Figure 2B), about 70\% proteins contained two or more peptides. With regard to the distribution of protein mass, most proteins had the molecular weight of over $10 \mathrm{kDa}$ at a broad range with favorable coverage (Figure 2C). Additionally, good sequence coverage was detected in most proteins, $33 \%$ and $56 \%$ of them constituted $20 \%$ and $>10 \%$ sequence coverage, respectively, which indicated great confidence (Figure 2D). Typically, those proteins that had the ratio of $>1.2$ (P-value of $<0.05$ ) were deemed to be up-regulated, while those that had the ratio of $<0.833$ (P-value of $<0.05$ ) were deemed as down-regulated. Based on the above-mentioned standards, a total of 731 DEPs were detected in CS and/or CT, including 161 up-regulated and 171 down-regulated DEPs in CS (Figure 3; Supplemental Table S1), as well as 193 up-regulated and 206 down-regulated DEPs in CT, separately (Figure 3; Supplemental Table S3). Of them, CS and CT shared 84 down-regulated and 79 up-regulated DEPs. Besides, there were 7 DEPs up-regulated in CT but down-regulated in CS, and 4 down-regulated in CT while up-regulated in CS.

\section{DEPs classification in CT and CS}

Altogether 2,575 proteins were detected. Specifically, proteins that had the P-value of $<0.05$ and fold change of $\geqq 1.2$ were deemed as DEPs. GO analysis was performed among 731 DEPs in CT and CS based on the above criteria (Supplemental Tables S3 and S4). 399 DEPs in CT were annotated, while such figure in CS was 332. These proteins were then examined with respect to molecular function (MF), biological process (BP), as well as cellular component (CC) through GO analysis (Figure 4). The results indicated that the binding and catalytic activities belonged to the dominant MFs; while defense response and responses to stimulus and stress were the predominant BPs; at the same time, cell part and extracellular region were the major CCs. These findings indicated that most DEPs took part in cellular process, responses to stimulus and stress, and catalytic activity, which suggested that cold stress had a marked effect on cell differentiation and physiological metabolism within peach cultivars with cold tolerance.

To examine those low-temperature-related metabolic pathways, 332 and 399 DEPs in CS and CT were additionally examined by KEGG analysis, separately. Of them, only those categories that were remarkably enriched and had the P-value of $<0.05$ were selected. According to our results, those CT-extracted DEPs were enriched into the biosynthesis of secondary metabolites (14.0\%), metabolic pathways $(25.1 \%)$, ribosome $(3.8 \%)$, carbon metabolism $(5.5 \%)$, peroxisome $(1.8 \%)$, starch and sucrose metabolism (3.0\%), and RNA transport (3.5\%), (Table 2). Those DEPs from CS were enriched into the carbon metabolism (4.2\%), secondary metabolites biosynthesis (12.1\%), metabolic pathways $(22.3 \%)$, peroxisome $(2.7 \%)$, RNA transport $(2.7 \%)$ and ribosome (6.6\%) in CS (Table 2). Supplemental Table S5 shows those DEPs within each KEGG category.

\section{RT-PCR was implemented to analyze the transcript expression of the as-screened DEPs}

Protein translation is frequently accompanied with phosphorylation, glycosylation and alternative splicing as a result, a gene may regulate multiple proteins, resulting in the inconsistency of gene expression with protein expression. To reveal the relationship of mRNA transcript level with protein abundance, RT-PCR was carried out to analyze the transcript levels of 10 screened DEPs. Five proteins (M5WUF0, 


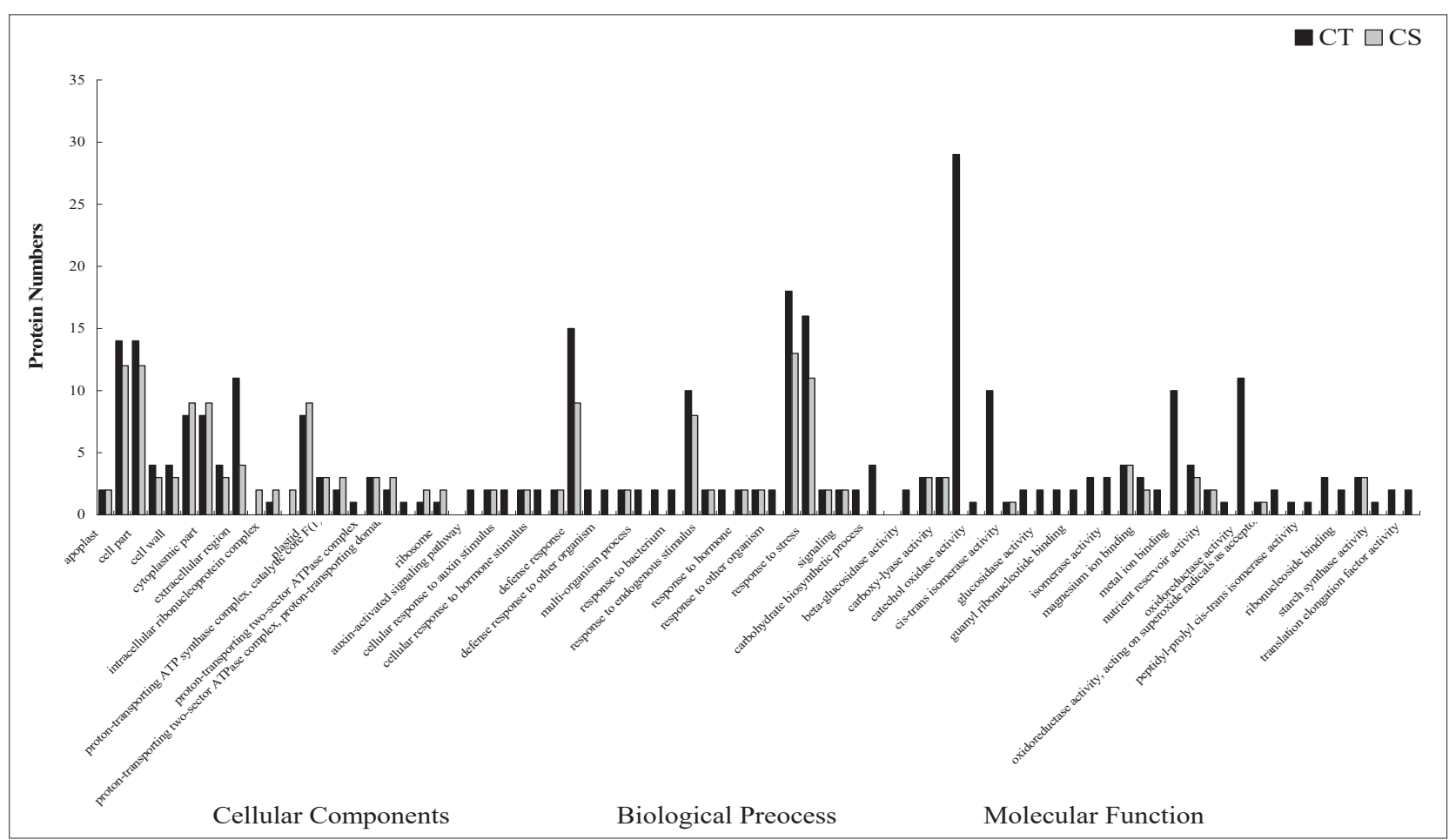

Figure 4. Gene Ontology (GO) annotations for DEGs within CT together with $\mathrm{CS}$ at $0^{\circ} \mathrm{C}$ and $-25^{\circ} \mathrm{C}$ for $48 \mathrm{~h}$, respectively. CT and CS represented the cold-tolerant and cold-sensitive peach cultivars, respectively.

M5W9D5, Q5QJB3, H9EBJ3, and M5W6Z4) were up-regulated in CT, but they remained unchanged in CS (Figure 5A-E). In addition, the M5WAW8 protein showed marked up-regulation within CS, but it was not changed within CT (Figure 5I). And the M5WC10 protein showed marked down-regulation within CS, but it was up-regulated within CT (Figure $5 \mathrm{H}$ ). Typically, one up-regulated (M5VVU6), together with one down-regulated (M5XCX2) proteins shared in CS and CT were screened (Figure 5G and 5F). Figure 5A-E suggests that five genes (M5WUF0, M5W9D5, Q5QJB3, H9EBJ3 and M5W6Z4) showed identical variation patterns at transcript level to those at the protein level. For the additional four genes, the protein and mRNA levels were different within CT or CS.

\section{Discussion}

This work aimed to examine the mechanisms of cold tolerance in cold-tolerant peach cultivars, and to offer relevant proteomic analysis, which might facilitate to breed peach cultivars with enhanced cold tolerance. The iTRAQ approach was adopted in the current research for proteomic analysis in the presence of various cold stress conditions to two peach cultivars. According to Figure 3, 399 and 332 proteins in CT and CS were detected, respectively. At the same time, there were more DEPs in CT than in CS (Figure 3). Based on the above analyses at the protein level, CT showed superior cold stress tolerance to CS. Besides, the cold stress mechanism was related to the regulation of numerous biological process-

TABLE 2. Main pathway enrichment analysis of DEPs.

\begin{tabular}{|c|c|c|c|}
\hline Pathway acc. & Pathway name & P-value & DEPs number \\
\hline \multicolumn{4}{|l|}{ CT } \\
\hline map00500 & Starch and sucrose metabolism & 0.0682425 & 12 \\
\hline map01100 & Metabolic pathways & 0.086388 & 100 \\
\hline map01110 & Biosynthesis of secondary metabolites & 0.1640456 & 56 \\
\hline map01200 & Carbon metabolism & 0.3499634 & 22 \\
\hline map03010 & Ribosome & 0.8617895 & 15 \\
\hline map03013 & RNA transport & 0.3171972 & 14 \\
\hline map04146 & Peroxisome & 0.2098524 & 7 \\
\hline \multicolumn{4}{|l|}{ CS } \\
\hline map01100 & Metabolic pathways & 0.4617484 & 74 \\
\hline map01110 & Biosynthesis of secondary metabolites & 0.5805352 & 40 \\
\hline map01200 & Carbon metabolism & 0.7894976 & 14 \\
\hline map03010 & Ribosome & 0.0531744 & 22 \\
\hline map03013 & RNA transport & 0.6830493 & 9 \\
\hline map04141 & Protein processing in endoplasmic reticulum & 0.6095607 & 12 \\
\hline map04146 & Peroxisome & 0.01495 & 9 \\
\hline
\end{tabular}



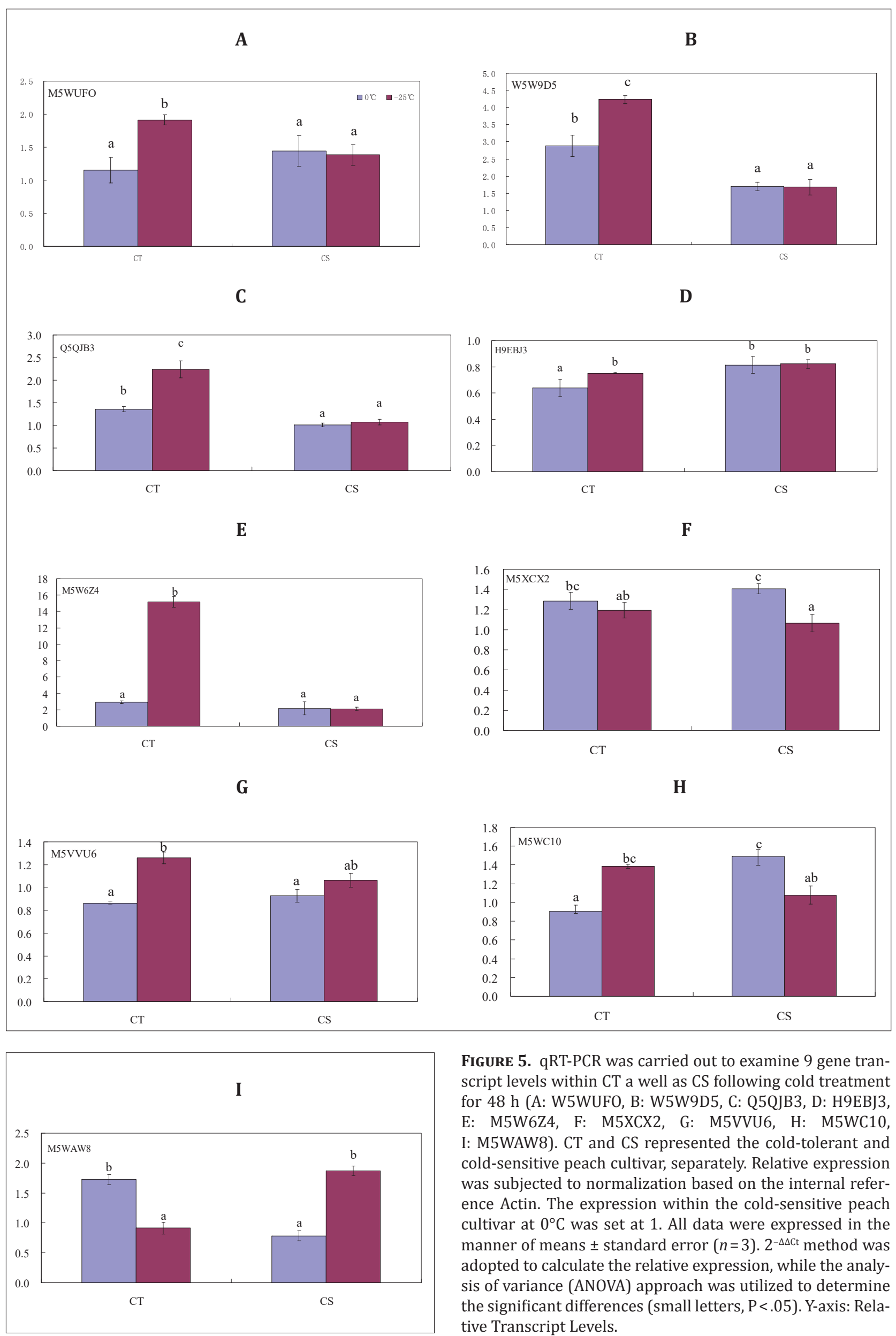

FIGURE 5. qRT-PCR was carried out to examine 9 gene transcript levels within CT a well as CS following cold treatment for 48 h (A: W5WUFO, B: W5W9D5, C: Q5QJB3, D: H9EBJ3, E: M5W6Z4, F: M5XCX2, G: M5VVU6, H: M5WC10, I: M5WAW8). CT and CS represented the cold-tolerant and cold-sensitive peach cultivar, separately. Relative expression was subjected to normalization based on the internal reference Actin. The expression within the cold-sensitive peach cultivar at $0^{\circ} \mathrm{C}$ was set at 1 . All data were expressed in the manner of means \pm standard error $(n=3) .2^{-\Delta \Delta \mathrm{Ct}}$ method was adopted to calculate the relative expression, while the analysis of variance (ANOVA) approach was utilized to determine the significant differences (small letters, $\mathrm{P}<.05$ ). Y-axis: Relative Transcript Levels. 
es. According to the bioinformatic analysis results, certain key DEPs were divided as several classes based on KEGG and GO analyses, as elaborated below.

\section{DEPs took part in carbon metabolism under cold stress}

As suggested by the proteomic analysis in this study, carbon metabolism exerted a vital part in responding to cold stress, which might be related to the effects of photosynthesis and respiration (Dong et al., 2018). Also, five carbohydrate proteins were identified (Supplemental Table S6). Substrates for synthesizing a variety of carbohydrates together with other metabolites are produced by the carbon metabolism pathway. In this study, the levels of several key enzymes involved in the pathway, including phosphoserine aminotransferase (PSAT, M1QDN1), glucose-6-phosphate isomerase (G6PI, M4QEK2), malic enzyme (ME, M5VWE8), glucose-6-phosphate 1-dehydrogenase (G6PD, M5XB81), serine hydroxymethyltransferase (SHMT, M5XDY2) and succinate dehydrogenase (SDH, A0A251Q7I8), were observed to be up-regulated (Supplemental Table S6) in CT, but they remained unchanged in CS. The up-regulated proteins observed within CT were probably related to the cold stress response. SDH has been identified as a key enzyme that participates in oxidative phosphorylation (OXPHOS) and tricarboxylic acid (TCA) cycle, and these two are the major metabolic pathways to generate ATP. Notably, energy deficiency in a plant is a major symptom in response to stress. Previous studies report that the higher SDH while lower REL contents contribute to maintaining the membrane integrity and reducing the lipid peroxidation in cold-tolerant chickpea (Karami-Moalem et al., 2018). In this study, REL in CT was lower than that in CS (Figure 1A), but GR of CT was higher than that of CS (Figure 1B), suggesting that REL might exert a vital part in cold stress tolerance of peach. The extent of REL shows a negative correlation with plant tolerance to stress. A high REL level results in a severe cold stress-induced damage (Peng et al., 2019). In addition, we identified that the Ribulose bisphosphate carboxylase large chain and small chain (A0A0A7NXG9, B5LBW7, H9CU12, M5XB14, M5XNQ1, and A0A1V0IT25) were down-regulated in CT or in CS (Supplemental Table S6), which was related to carbon fixation and carbon metabolism in photosynthetic microorganisms. Such results conformed to one prior study on winter turnip rape (freezing-tolerant) (Xu et al., 2018). A plant that is subjected to continuous abiotic and biotic stresses will result in protein degradation, implying that cold stress inhibits protein synthesis.

\section{DEPs were involved in phenylpropanoid biosynthesis under cold stress conditions}

Critically, the phenylpropanoid pathway is involved in the plant tolerance to stresses, which plays a role as the natural factory for producing various secondary metabolites to respond to abiotic and biotic stimulation (Deng and Lu, 2017). Phenylpropanoids are the secondary metabolites generated by phenylalanine (Bonawitz and Chapple, 2010; Vanholme et al., 2012, 2013). In this study, two peroxidases (PODs, including M5WUF0 and M5W9D5) related to the biosynthesis of phenylpropanoid were detected (Supplemental Table S6). According to our results, these two PODs were more abundant in CT than in CS (Supplemental Table S6), which suggested their important parts in maintaining homeostasis and preventing the lethal effects of reactive oxygen species (ROS) (Foyer and Noctor, 2005). Under cold stress conditions, PODs, which are the osmotic substances that exert vital roles in adjusting osmotic pressure, are accumulated rapidly (Xu et al., 2018). Similarly, PODs are revealed in a prior proteomic study to be highly expressed in pumpkin rootstock-grafted watermelon seedlings under cold stress conditions (Shi et al., 2019). Generally, stress is accompanying with ROS accumulation in plant cells, including the hydrogen peroxide $\left(\mathrm{H}_{2} \mathrm{O}_{2}\right)$, hydroxyl radical $\left(\mathrm{OH}^{\circ}\right)$, and superoxide radical $\left(\mathrm{O}_{2}^{-}\right)$. ROS generation is an initial cold stress response in Arabidopsis and rice (Fanucchi et al., 2012; Lee et al., 2007), and such ROS attacks macromolecules and induces membrane damage. Nonetheless, there are adaptive strategies in plants against ROS, such as activation of the non-enzymatic and enzymatic antioxidant defense systems (Sytar et al., 2013). For example, in trifoliate orange (Poncirus trifoliata), PtrERF109 is bound to peroxidase (POD)-coding gene PtrPrx1 promoter directly for modulating the homeostasis of ROS, which thereby positively responds to cold stress (Wang et al., 2018). In this study, RT-PCR analysis also showed that the transcription levels of two genes encoding PODs (M5WUF0 and M5W9D5) were consistent with the results of iTRAQ analysis (Figure 5A-B). PODs are also the critical components in the phenylpropanoid biosynthesis pathway, which contribute to catalyzing phenylpropanoids for the biosynthesis of flavonoids (Royo et al., 2019). Further, flavonoids are the secondary metabolite in plants, which exert various pharmacological and biological effects, such as protection from abiotic stress in plants (Peng et al., 2019). In this study, two proteins (A0A0K1TPE2 and H9EBJ3) related to flavonoid biosynthesis were identified (Supplemental Table S6). Anthocyanidin reductase and flavonoid 3' hydroxylase were up-regulated in CT at cold stress conditions, but they remained unchanged in CS, which conformed to a prior work regarding Ammopiptanthus mongolicus under drought and cold stress (Wu et al., 2014). Additionally, the level of flavonoid biosynthesis protein (H9EBJ3) was consistent with its mRNA level (Figure 5D), which suggested that there was modulation at post-transcriptional level under cold stress conditions for CT. For a long time, flavonoids are identified to be the major plant antioxidants that facilitate to protect from cell apoptosis under oxidative stress (OS) and environmental stress.

\section{DEPs took part in sucrose and starch metabolism under cold stress}

Many proteins involved in sucrose and starch metabolism were changed to respond to cold stress within CT and CS. It is well-known that soluble sugars (sucrose, glucose, fructose, and trehalose) may serve as the cryoprotectant agents for protecting tissue metabolism, which is achieved through preserving organelle and cell membrane integrity, thus improving the plant tissue tolerance to cold stress (Bhandari and Nayyar, 2014). In this study, a glucose-6-phosphate isomerase (M4QEK2), a glucose-1-phosphate adenylyltransferase (M5WU36), and an alpha-1,4 glucan phosphorylase (M5WK36, M5WYM2) were also identified to participate in sucrose and starch metabolism. These proteins were up-regulated within CT but remained unchanged (M4QEK2 and M5WK36) or decreased (M5WU36) in CS under cold stress (Supplemental Table S6), which indicated that cold-resistant cultivars accumulated more soluble sugars under cold stress. Additionally, two amylases, namely, alpha-amylase (M5VVU6) and starch synthase (M5X7A6), were identified. The abundance of alpha-amylase was lower in CT (ratio: 0.67) than in CS (ratio: 0.78), but glucose-1-phosphate adenylyltransferase (G1PA, M5WU36) was higher (ratio: 1.35) in CT than CS (ratio: 1.21) under cold stress. G1PA catalyz- 
es the synthesis of the activated glucose donor, adenosine diphosphate glucose (ADPG) from glucose-1-phosphate (Glc-1-P) and ATP. Glucose, as a soluble monosaccharide, is an osmotic substance that can improve the water retention ability of cells under cold stress (Wu et al., 2016). Thus, it was speculated that the degradation of polysaccharides to soluble monosaccharide might be faster in CT than in CS in response to cold stress. Starch synthase was up-regulated in CS but had no change in CT, indicating that the conversion efficiency of monosaccharide in CS might be low, resulting in starch synthase accumulation. Our results conformed to a prior study on strawberry in the presence of cold stress (Zhang et al., 2019).

\section{DEPs were involved in defense response under cold stress}

PR protein synthesis is an important plant defense characteristic to respond to the non-abiotic and/or abiotic stress conditions. Among these defense responses, the production of PR proteins is well documented. For example, the over-expressed PR protein-encoding genes boost the tolerance to pathogens and heavy metal stresses (Sarowar et al., 2005), drought stress environments in tobacco (Jain et al., 2012), and salt stress in Halogeton glomeratus (Wang et al., 2016b). This proteomic study identified three pathogenesis-related (PR2, 5, 10) proteins (G9CM54, F8RNZ5, and B5KVN9, Supplemental Table S6), which were involved in homologous recombination and RNA transport. Of them, PR2 and PR5 proteins were present exclusively in CT, but they were not detected in CS under cold stress. However, PR10 protein was present exclusively in CS but not detected in CT. A previous study has proven that cold stress activates the innate immune responses through up-regulating the expression of the salicylic acid (SA) pathway marker genes, namely, PR2 and PR5, in Arabidopsis thaliana (Wu et al., 2019). This result indicated the presence of various strategies within sensitive and tolerant peach species to respond to cold stress.

\section{DEPs were involved in ribosome proteins at cold stress conditions}

Ribosome is the protein biosynthesis site (Xia et al., 2016), which shows high sensitivity to any environment change; typically, a subtle change in temperature rapidly while temporarily represses the genes of ribosome protein (RPs) subunits, thus impacting the ribosomal proteins (Warner, 1999). With regard to this study, 15 and 22 DEPs were considerably enriched in CT and CS. Besides, two ribosomal proteins (A0A1V0IQ99 and M5VRV9) and one hydroxyproline-rich glycolprotein (H2EIF3) were also identified (Supplemental Table S6). These three proteins were down-regulated within CS in cold stress conditions, but remained unchanged within $\mathrm{CT}$, which suggested the presence of the steady environment response in CT at low-temperature conditions, assisting the plants in resisting the adverse stress. Such a result was not entirely consistent with the previous study suggesting that the ribosomal protein was up-regulated in Flammulina velutipes and winter turnip rape under cold stress (Liu et al., 2017; Zeng et al., 2018). Few existing studies are conducted to examine the direct relationship of cold stress with RPs, but the reduced RPs levels observed in CS must also be taken into consideration. RPs were speculated to take part in the response to stress as the regulatory components in CS, even though more efforts are required to elaborate on the regulatory mechanism. In addition, harpin binding protein 1 (HB1, Q5QJB3) was also identified (Supplemental Table
S6). In this study, HB1 was up-regulated in CT but remained unchanged in CS. On the other hand, HBs produced via the gram-negative bacteria pathogenic to plants trigger various defenses in plants to resist diverse pathogens. As reported by one prior study, harpin proteins enhanced insect and disease tolerance of crop plants (Tian et al., 2015). Further analysis also showed that the transcription level of HB1 (Q5QJB3) was consistent with those proteomic data (Figure 5C). The over-expressed harpin-encoding genes remarkably boost the tolerance within soybean, which is achieved through inducing the defense signal transduction pathways-mediated tolerance responses (Tian et al., 2015). Such a result indicates the vital part of this protein in cold stress tolerance in peach.

\section{DEPs were involved in Carbonic anhydrase under cold stress}

Carbonic anhydrases (CAs) function to catalyze the interconversion between $\mathrm{HCO}_{3}$ - and $\mathrm{CO}_{2}$ (Dimario et al., 2017). Several CA families exist in nature, which are designated as CA $\alpha-\theta$ (Dimario et al., 2018). Interestingly, M5W6Z4 (Supplemental Table S2), which is extensively distributed in photosynthetic bacteria, algae, and plants, exerts a vital part in $\mathrm{CO}_{2}$ concentrating mechanisms (CCMs) to boost $\mathrm{CO}_{2}$ delivery to Rubisco (Wang et al., 2016a). In this study, CA was up-regulated in CT (mean fold change of 2.8) but remained unchanged in CS under cold stress. The CA activity is detected in Brassica napus (the cultivar 2AF009 with high drought tolerance) (Wang et al., 2016c). Such findings indicated that the CA identified within CT might exert a vital part in responding to cold stress. Furthermore, Rubisco (A0A0A7NXG9, B5LBW7, H9CU12, M5XB14, M5XNQ1, and A0A1V0IT25), which was constituted by 8 small and 8 large subunits, respectively, was down-regulated in CT or CS (Supplemental Tables S1 and S2). Further analysis also showed that the transcriptional expression of Ribulose bisphosphate carboxylase small chain (M5XNQ1) showed no significant difference between CS and CT (Supplemental Table S6), and such results conformed to those obtained from iTRAQ data. Therefore, it was speculated that the process from $\mathrm{CO}_{2}$ to $\mathrm{Ru}$ bisco might be inhibited, which resulted in CA accumulation. Additionally, the transcriptional analysis on CA (M5W6Z4) was performed by RT-PCR, which suggested that its gene expression showed a consistent pattern with the protein level (Figure 5E). Herein, this observation suggested that CA might be an important implication for evaluating the cold tolerance ability, but further study was warranted.

\section{Conclusions}

Low temperature is a critical limiting factor for the growth, development, and distribution of peach. Proteomic analysis can offer tremendous data regarding individual proteins taking part during certain biological responses. Comparative proteomic analysis in this study suggested that the cold tolerance mechanisms in peach were achieved through energy generated during phenylpropanoid biosynthesis, sucrose and starch metabolism, and carbon metabolism. Besides, there were more DEPs within CT than those within CS. Notably, peroxidase, flavonoid, carbonic anhydrase, and harpin proteins might positively regulate the cold stress response in CT. In addition, the different PR proteins suggested the presence of various strategies for cold stress resistance in CT as well as in CS. Besides, the reliability of iTRAQ findings was validated through experiments on the physiological indicators and six genes at the transcript level. As suggested by the above findings, applying proteomics in combination 
with bioinformatics analysis serves as an efficient tool to examine the underlying mechanisms of cold tolerance in peach at the molecular level.

\section{Acknowledgments}

This work was supported by the Fundamental Research Funds for Hebei Academy of Agriculture and Forestry Sciences (Nos. 2018020301, C19C07-0402, 2019-3-4-4 and 2019-3-5-1-02), China Agriculture Research System of MOF and MARA (No. CARS-30-Z-02) and the Hebei Province Key Research and Development Project (No. 17226341).

\section{Conflict of interest}

The authors declare that they have no conflict of interest.

\section{References}

Bhandari, K., and Nayyar, H. (2014). Low temperature stress in plants: An overview of roles of cryoprotectants in defense. Physiolog. Mech. Adapt. Strat. in Plants Under Changing Environm., p. 193-265. https://doi.org/10.1007/978-1-4614-8591-9_9.

Bonawitz, N.D., and Chapple, C. (2010). The genetics of lignin biosynthesis: Connecting genotype to phenotype. Ann. Rev. Gen. 44, 337-363. https://doi.org/10.1146/annurev-genet-102209-163508.

Chen, T.T., Zhang, L., Shang, H.H., Liu, S.D., Peng, J., Gong, W.K., Shi, Y.Z., Zhang, S.P., Li, J.W., Gong, J.W., Ge, Q., Liu, A.Y., Ma, H.J., Zhao, X.H., and Yuan, Y.L. (2016). iTRAQ-based quantitative proteomic analysis of cotton roots and leaves reveals pathways associated with salt stress. PloS ONE 11(2), e0148487. https://doi.org/10.1371/journal. pone. 0148487

Chen, Z., Wen, B., Wang, Q.H., Wei, T., Guo, W.J., Bai, X., Zhao, J.J., Sun, Y., Tang, Q., Lin, Z.L., Lin, L., and Liu, S.Q. (2013). Quantitative proteomics reveals the temperature-dependent proteins encoded by a series of cluster genes in Thermoanaerobacter tengcongensis. Mol. Cell. Proteomics 12(8), 2266-2277. https://doi.org/10.1074/mcp. M112.025817.

Chu, P., Yan, G.X., Yang, Q., Zhai, L.N., Zhang, C., Zhang, F.Q., and Guan, R.Z. (2015). iTRAQ-based quantitative proteomics analysis of Brassica napus leaves reveals pathways associated with chlorophyll deficiency. J. Proteomics 113, 244-259. https://doi.org/10.1016/j. jprot.2014.10.005.

Conesa, A., Götz, S., García-Gómez, J.M., Terol, J., Talón, M., and Robles, M. (2005). Blast2GO: A universal tool for annotation, visualization and analysis in functional genomics research. Bioinformatics 21, 3674-3676. https://doi.org/10.1093/bioinformatics/bti610.

Deng, J., Yin, X.J., Xiang, Y., Xin, H.P., Li, S.H., and Yang, P.F. (2017). iTRAQ-based comparative proteomic analyses of two grapevine cultivars in response to cold stress. Current Proteomics 14, 42-52. https://doi.org/10.2174/1570164613666161130124819.

Deng, Y., and Lu, S. (2017). Biosynthesis and regulation of phenylpropanoids in plants. Critical Rev. in Plant Sci. 36(4), 257290. https://doi.org/10.1080/07352689.2017.1402852.

Dimario, R.J., Clayton, H., Mukherjee, A., Ludwig, M., and Moroney, J.V. (2017). Plant carbonic anhydrases: structures, locations, evolution, and physiological roles. Molecular Plant 10, 30-46. https://doi. org/10.1016/j.molp.2016.09.001.

Dimario, R.J., Machingura, M.C., Waldrop, G.L., and Moroney, J.V. (2018). The many types of carbonic anhydrases in photosynthetic organisms. Plant Sci. 268,11-17. https://doi.org/10.1016/j.plantsci.2017.12.002.

Dong, F., Shi, Y., Liu, M., Fan, K., Zhang, Q., and Ruan, J. (2018). iTRAQbased quantitative proteomics analysis reveals the mechanism underlying the weakening of carbon metabolism in chlorotic tea leaves. Int. J. Molec. Sci. 19(12), 3943. https://doi.org/10.3390/ ijms19123943.
Fan, H.F., Xu, Y.L., Du, C.X., and Wu, X. (2015). Phloem sap proteome studied by iTRAQ provides integrated insight into salinity response mechanisms in cucumber plants. J. Proteomics 125, 54-67.https:// doi.org/10.1016/j.jprot.2015.05.001.

Fanucchi, F., Alpi, E., Olivieri, S., Cannistraci, C.V., Bachi, A., Alpi, A., and Alessio, M. (2012). Acclimation increases freezing stress response of Arabidopsis thaliana at proteome level. Biochem. Biophys. Acta 1824, 813-825. https://doi.org/10.1016/j.bbapap.2012.03.015.

Foyer, C.H., and Noctor, G. (2005). Redox homeostasis and antioxidant signaling: A metabolic interface between stress perception and physiological responses. Plant Cell 17(7), 1866-1875. https://doi. org/10.1105/tpc.105.033589.

Howell, G.S., Flore, J.A., and Miller, D.P. (1997). Cultivar, rootstock and twig portion affect cold resistance of peach (Prunus persica (L.) Batch). Adv. Hortic. Sci. 11(1), 30-36.

Jain, S., Kumar, D., Jain, M., Chaudhary, P., Deswal, R., and Sarin, N.B. (2012). Ectopic overexpression of a salt stress-induced pathogenesisrelated class 10 protein (PR10) gene from peanut (Arachis hypogaea L.) affords broad spectrum abiotic stress tolerance in transgenic tobacco. Plant Cell Tiss. Org. 109, 19-31. https://doi.org/10.1007/ s11240-011-0069-6.

Kanehisa, M., Araki, M., Goto, S., Hattori, M., Hirakawa, M., Itoh, M., Katayama, T., Kawashima, S., Okuda, S., Tokimatsu, T., and Yamanishi, Y. (2008). KEGG for linking genomes to life and the environment. Nucleic Acids Res. 36, 480-484. https://doi.org/10.1093/nar/ gkm882.

Karami-Moalem, S., Maali-Amiri, R., and Kazemi-Shahandashti, S.S. (2018). Effect of cold stress on oxidative damage and mitochondrial respiratory properties in chickpea. Plant Physiol. Biochem. 122, 3139. https://doi.org/10.1016/j.plaphy.2017.11.011.

Lee, D.G., Ahsan, N., Lee, S.H., Kang, K.Y., Lee, J.J., and Lee, B.H. (2007). An approach to identify cold-induced low-abundant proteins in rice leaf. CR Biol. 330, 215-225. https://doi.org/10.1016/j. crvi.2007.01.001.

Li, B., Ning, L., Zhang, J., Bao, M., and Zhang, W. (2015). Transcriptional profiling of Petunia seedlings reveals candidate regulators of the cold stress response. Front. Plant Sci. 6, 118. https://doi.org/10.3389/ fpls.2015.00118.

Liu, J., Pang, C., Wei, H., Song, M., Meng, Y., Ma, J., Fan, S., and Yu, S.X. (2015). iTRAQ-facilitated proteomic profiling of anthers from a photosensitive male sterile mutant and wild-type cotton (Gossypium hirsutum L.). J. Proteomics 126, 68-81. https://doi.org/10.1016/j. jprot.2015.05.031.

Liu, J.Y., Men, J.L., Chang, M.C., Feng, C.P., and Yuan, L.G. (2017). iTRAQ-based quantitative proteome revealed metabolic changes of Flammulina velutipes mycelia in response to cold stress. J. Proteomics 156, 75-84. https://doi.org/10.1016/j.jprot.2017.01.009.

Marafon, A.C., Herter, F.G., Bacarin, M.A., and Hawerroth, F.J. (2009). Atividade da peroxidase durante o período hibernal de plantas de pessegueiro (Prunus persica (L.) Batsch.) cv. Jubileu com e sem sintomas da morte precoce. Rev. Brasil. Fruticult. 31(4), 938-942. https://doi.org/10.1590/S0100-29452009000400004.

Nilo-Poyanco, R., Vizoso, P., Sanhueza, D., Balic, I., Meneses, C., Orellana, A., and Campos-Vargas, R. (2019). A Prunus persica genome-wide RNA-seq approach uncovers major differences in the transcriptome among chilling injury sensitive and non-sensitive varieties. Physiol. Plant. 166(3), 772-793. https://doi.org/10.1111/ ppl.12831.

Niu, R.X., Zhao, X.M., Wang, C.B., and Wang, F.L. (2020). Transcriptome profiling of Prunus persica branches reveals candidate genes potentially involved in freezing tolerance. Sci. Hortic. 259, 108775. https://doi.org/10.1016/j.scienta.2019.108775. 
Peng, X., Wu, H., Chen, H.J., Zhang, Y.J., Qiu, D., and Zhang, Z.Y. (2019). Transcriptome profiling reveals candidate flavonol-related genes of Tetrastigma hemsleyanum under cold stress. BMC Genomics 20(1), 687. https://doi.org/10.1186/s12864-019-6045-y.

Royo, B., Esteban, R., Buezo, J., Santamaria, E., Fernández-Irigoyen, J., Becker, D., and Moran, J.F. (2019). The proteome of Medicago truncatula in response to ammonium and urea nutrition reveals the role of membrane proteins and enzymes of root lignification. Environm. Experim. Botany 162, 168-180. https://doi.org/10.1016/j. envexpbot.2019.02.010.

Saravanan, R.S., and Rose, J.K.C. (2004). A critical evaluation of sample extraction techniques for enhanced proteomic analysis of recalcitrant plant tissues. Proteomics 4, 2522-2532. https://doi. org/10.1002/pmic.200300789.

Sarowar, S., Kim, Y.J., Kim, E.N., Kim, K.D., Hwang, B.K., Islam, R., and Shin, J.S. (2005). Overexpression of a pepper basic pathogenesisrelated protein 1 gene in tobacco plants enhances resistance to heavy metal and pathogen stresses. Plant Cell Rep. 24, 216-224. https:// doi.org/10.1007/s00299-005-0928-x.

Shi, X.F., Wang, X.B., Cheng, F., Cao, H.S., Liang, H., Lu, J.Y., Kong, Q.S., and Bie, Z.L. (2019). iTRAQ-based quantitative proteomics analysis of cold stress-induced mechanisms in grafted watermelon seedlings. J. Proteomics 192, 311-320. https://doi.org/10.1016/j. jprot.2018.09.012.

Su, Y.C., Xu, L.P., Wang, Z.Q., Peng, Q., Yang, Y.T., Chen, Y., and Que, Y.X (2016). Comparative proteomics reveals that central metabolism changes are associated with resistance against Sporisorium scitamineum in sugarcane. BMC Genomics 17(1), 800. https://doi. org/10.1186/s12864-016-3146-8.

Sytar, O., Kumar, A., Latowski, D., Kuczynska, P., Strzałka, K., and Prasad, M. (2013). Heavy metal-induced oxidative damage, defense reactions, and detoxification mechanisms in plants. Acta Physiol Plant. 35, 985-999. https://doi.org/10.1007/s11738-012-1169-6.

Tareen, M.J., Singh, Z., Khan, A.S., Abbasi, N.A., and Naveed, M. (2017). Combined applications of aminoethoxyvinylglycine with salicylic acid or nitric oxide reduce oxidative stress in peach during ripening and cold storage. J. Plant Growth Regul. 36(4), 983-994. https://doi. org/10.1007/s00344-017-9702-x.

Tian, X., Liu, Y., Huang, Z.G., Duan, H.P., Tong, J.H., He, X.L., Gu, W.H., Ma, H., and Xiao, L.G. (2015). Comparative proteomic analysis of seedling leaves of cold-tolerant and -sensitive spring soybean cultivars. Mol. Biol. Rep. 42(3), 581-601. https://doi.org/10.1007/ s11033-014-3803-4.

Vanholme, R., Storme, V., Vanholme, B., Sundin, L., Christensen, J.H., Goeminne, G., Halpin, C., Rohde, A., Morreel, K., and Boerjan, W. (2012). A systems biology view of responses to lignin biosynthesis perturbations in Arabidopsis. Plant Cell. 24, 3506-3529. https://doi. org/10.1105/tpc.112.102574.

Vanholme, R., Cesarino, I., Rataj, K., Xiao, Y.G., Sundin, L., Goeminne, G., Hoon, K., Cross, J., Morreel, K., Araujo, P., Welsh, L., Haustraete, J., McClellan, C., Vanholme, B., Ralph, J., Simpson Gordon, G., Halpin, C., and Boerjan, W. (2013). Caffeoyl shikimate esterase (CSE) is an enzyme in the lignin biosynthetic pathway in Arabidopsis. Science 341, 1103-1106. https://doi.org/10.1126/science.1241602.

Wang, J., Wang, X.R., Zhou, Q., Yang, J.M., Guo, H.X., Yang, L.J., and Liu, W.Q. (2016a). iTRAQ protein profile analysis provides integrated insight into mechanisms of tolerance to TMV in tobacco (Nicotiana tabacum). J. Proteomics 132, 21-30. https://doi.org/10.1016/j. jprot.2015.11.009.

Wang, J.C., Yao, L., Li, B.C., Meng, Y.Y., Ma, X.L., Lai, Y., Si, E.J., Ren, P.R., Yang K., Shang, X.W., and Wang, H.J. (2016b). Comparative proteomic analysis of cultured suspension cells of the halophyte Halogeton glomeratus by iTRAQ provides insights into response mechanisms to salt stress. Front. Plant Sci. 7, 110. https://doi.org/10.3389/fpls.2016.00110.
Wang, L.M., Jin, X., Li, Q.B., Wang, X.C., Li, Z.Y., and Wu, X.M. (2016c). Comparative proteomics reveals that phosphorylation of $\beta$-carbonic anhydrase 1 might be important for adaptation to drought stress drought in Brassica napus. Sci. Rep. 6, 39024. https://doi. org/10.1038/srep39024

Wang, M., Dai, W., Du, J., Ming, R., Dahro, B., and Liu, J.H. (2018). ERF109 of trifoliate orange (Poncirus trifoliata (L.) Raf.) contributes to cold tolerance by directly regulating expression of Prx1 involved in antioxidative process. Plant Biotechnol. J. 17(7), 1316-1332. https://doi.org/10.1111/pbi.13056

Wang, W., Vignani, R., Scali, M., and Cresti, M. (2006). A universal and rapid protocol for protein extraction from recalcitrant plant tissues for proteomic analysis. Electrophoresis 27(13), 2782-2786. https:// doi.org/10.1002/elps.200500722.

Warner, J.R. (1999). The economics of ribosome biosynthesis in yeast. Trends Biochem. Sci. 24, 437-440. https://doi.org/10.1016/ S0968-0004(99)01460-7.

Wu, X., Yuan, J., Luo, A., Chen, Y., and Fan, Y. (2016). Drought stress and re-watering increase secondary metabolites and enzyme activity in Dendrobium moniliforme. Ind. Crop. Prod. 94, 385-393. https://doi. org/10.1016/j.indcrop.2016.08.041.

Wu, X.Q., Mason, A.M., Yu, M.L., Ma, R.J., and Yu, Z.F. (2017) Quantitative proteomic analysis of pre- and post-harvest peach fruit ripening based on iTRAQ technique. Acta Physiol. Plant. 39, 181. https://doi.org/10.1007/s11738-017-2478-6.

Wu, Y.Q., Wei, W., Pang, X.Y., Wang, X.F., Zhang, H.L., Dong, B., Xing, Y.P., Li, X.G., and Wang, M.Y. (2014). Comparative transcriptome profiling of a desert evergreen shrub, Ammopiptanthus mongolicus, in response to drought and cold stresses. BMC Genomics 15(1), 671. https://doi.org/10.1186/1471-2164-15-671.

Wu, Z., Han, S., Zhou, H., Tuang, Z.K., Wang, Y., Jin, Y., Shi, H., and Yang, W. (2019). Cold stress activates disease resistance in Arabidopsis thaliana through a salicylic acid dependent pathway. Plant Cell Environm. 42(9), 2645-2663. https://doi.org/10.1111/pce.13579.

Xia, K., Zang, N., Zhang, J., Zhang, H., Li, Y., Liu, Y., Feng, W., and Liang, $X$. (2016). New insights into the mechanisms of acetic acid resistance in Acetobacter pasteurianus using iTRAQ-dependent quantitative proteomic analysis. Int. J. Food Microbiol. 238, 241-251. https://doi. org/10.1016/j.ijfoodmicro.2016.09.016

Xu, Y.Z., Zeng, X.C., Wu, J., Zhang, F.Q Li, C.X., Jiang, J.J., Wang, Y.P. and Sun, W.C. (2018). iTRAQ-based quantitative proteome revealed metabolic changes in winter turnip rape (Brassica rapa L.) under cold stress. Int. J. Mol. Sci. 19, 3346. https://doi.org/10.3390/ ijms19113346.

Yao, Y., Yang, Y.W., and Liu, J.Y. (2006). An efficient protein preparation for proteomic analysis of developing cotton fibers by 2-DE. Electrophoresis 27(22), 4559-4569. https://doi.org/10.1002/ elps. 200600111.

Yun, S.K., Bae, H.J., Chung, K.H., Yoon, I.K., Nam, E.Y., Kwon, J.H., and Jun, J.H. (2014). Sugar, starch, and proline in peach trees exposed to freezing temperatures during dehardening. Agric. Sci. 5(10), 913921. https://doi.org/10.4236/as.2014.510099.

Zeng, X.C., Xu, Y.Z., Jiang, J.J., Zhang, F.Q., Ma, L., Wu, D.W., Wang, Y.P., and Sun, W.C. (2018). Identification of cold stress responsive microRNAs in two winter turnip rape (Brassica rapa L.) by high throughput sequencing. BMC Plant Biol. 18, 52-64. https://doi. org/10.1186/s12870-018-1242-4

Zhang, N., Zhang, L.G., Shi, C.N., Zhao, L., Cui, D.Q., and Chen, F. (2018). Identification of proteins using iTRAQ and virus-induced gene silencing reveals three bread wheat proteins involved in the response to combined osmotic-cold stress. J. Proteome Res. 6, 22562281. https://doi.org/10.1021/acs.jproteome.7b00745 
Zhang, Y., Zhang, Y.T., Lin, Y.X., Luo, Y., Wang, X.R., Chen, Q., Sun, B., Wang, Y., Li, M.Y., and Tang, H.R. (2019). A transcriptomic analysis reveals diverse regulatory networks that respond to cold stress in strawberry (Fragaria $\times$ ananassa). Int. J. Genomics 7106092. https:// doi.org/10.1155/2019/7106092.

Zhang. W., Zhang, H.L., Ning, L., Li, Y.B., and Bao, M.Z. (2016). Quantitative proteomic analysis provides novel insights into cold stress responses in Petunia seedlings. Front. Plant Sci. 7, 136. https://doi.org/10.3389/fpls.2016.00136.

Received: Jan. 29, 2020

Accepted: Jul. 28, 2020

Addresses of authors:

Yonghong $\mathrm{Li}^{1, *}$, Zhaoyuan Wang ${ }^{1}$, Qihang Tian ${ }^{1}$,

Yuchao Zhou ${ }^{2}$, Jintao Xu ${ }^{1}$, Ruifeng Chang ${ }^{1}$, Hu Chen ${ }^{1}$ and Guojian Liu ${ }^{1}$

${ }^{1}$ Changli Fruit Institute, Hebei Academy of Agriculture and Forestry Sciences, Changli, Hebei 066600, PR China

${ }^{2}$ Hebei Academy of Agriculture and Forestry Sciences,

Shijiazhuang, Hebei 050051, PR China

* Corresponding author;

E-mail: liyonghongpeng@126.com

SUPPLEMENTAL INFORMATION - TABLES S1 -S6:

See www.ishs.org./eJHS. 